Journal of Zhejiang University-SCIENCE B (Biomedicine \& Biotechnology) ISSN 1673-1581 (Print); ISSN 1862-1783 (Online)

www.jzus.zju.edu.cn; www.springerlink.com

E-mail: jzus@zju.edu.cn

\title{
Correspondence:
}

\section{Clinical value of the emergency department in screening and diagnosis of COVID-19 in China*}

\author{
Qin $Z_{H A N G}{ }^{1,2}$, Jian PAN $^{1,2}$, Min-xing $Z_{H A O}{ }^{1,2}$, \\ Yuan-qiang $\mathrm{LU}^{\dagger \$ 1,2}$ \\ ${ }^{1}$ Department of Emergency Medicine, the First Affiliated Hospital, \\ School of Medicine, Zhejiang University, Hangzhou 310003, China \\ ${ }^{2}$ Key Laboratory for Diagnosis and Treatment of Aging and \\ Physic-chemical Injury Diseases of Zhejiang Province, the First \\ Affiliated Hospital, School of Medicine, Zhejiang University, \\ Hangzhou 310003, China \\ †E-mail: luyuanqiang@zju.edu.cn
}

https://doi.org/10.1631/jzus.B2010011

Published online Mar. 16, 2020

Since the global outbreak of severe acute respiratory syndrome (SARS) in 2003, China has gradually built a robust prevention and control system for sudden infectious diseases. All large hospitals have a fever clinic that isolates patients with all kinds of acute communicable diseases as the first line of medical defense. The emergency department, as the second line of medical defense in hospitals, is constantly shouldering the heavy responsibility of screening communicable diseases while also treating all kinds of other non-communicable acute and critical diseases (Zhang et al., 2012; Zhu et al., 2015; Wang et al., 2017; Feng et al., 2018; Lu, 2018; Xu and Lu, 2019). An outbreak of pneumonia of unknown etiology that began in Wuhan city (China) has spread rapidly in China since December 2019 (Huang et al., 2020; WHO, 2020; Zhu et al., 2020). In February 2020, the National Health Commission of China named the disease a novel coronavirus pneumonia $(\mathrm{NCP})$; then, it was formally named the coronavirus

\footnotetext{
${ }^{\ddagger}$ Corresponding author

* Project supported by the Foundation of Key Discipline Construction of Zhejiang Province for Traditional Chinese Medicine (No. $2017-$ XK-A36), China

(DD ORCID: Yuan-qiang LU, https://orcid.org/0000-0002-9057-4344 (C) Zhejiang University and Springer-Verlag GmbH Germany, part of Springer Nature 2020
}

disease 2019 (COVID-19) by the World Health Organization (WHO) on Feb. 11, 2020. The Coronavirus Study Group of the International Committee on Taxonomy of Viruses designated this causative virus as SARS coronavirus 2 (SARS-CoV-2). SARS-CoV-2 belongs to the $\beta$ coronavirus genus, and its pathogenic mechanism has not been clarified, which requires further study. To better understand the clinical characteristics of COVID-19 and more effectively prevent and control this disease, we retrospectively analyzed four representative cases of COVID-19 that had recently been screened and diagnosed in our emergency department.

\section{Clinical data}

Case 1: A 40-year-old woman began to appear feverish $4 \mathrm{~d}$ prior to diagnosis, with the highest body temperature of $38.5^{\circ} \mathrm{C}$, accompanied by sore throat, joint soreness, and occasionally dry cough. No sputum, chills, chest tightness, or other discomforts were observed. Her body temperature returned to normal over the next $2 \mathrm{~d}$, but she still had a dry cough and sore throat. Later, she visited the emergency department of our hospital on Jan. 21, 2020, denying a history of living in or traveling to the epidemic areas and denying exposure to other patients with COVID-19 over the past month. The results of the physical exam and laboratory tests for this patient upon admission are shown in Table 1 . The physical exam revealed no dry or moist rales in the bilateral lungs. The result of the influenza A+B RNA assay (real-time reverse transcription-polymerase chain reaction (rRT-PCR)) was negative. A lung computed tomography (CT) scan showed a nodular dense shadow in the subpleural basal segment of the right lower lobe with a halo sign, small patchy ground-glass shadows under the pleura in the right lower lung base section, and patchy 
Table 1 Characteristic results of the patients with coronavirus disease 2019 (COVID-19) that underwent physical examination and laboratory investigation upon admission

\begin{tabular}{ccccccccc}
\hline Patient & $T\left({ }^{\circ} \mathrm{C}\right)$ & $P($ beats $/ \mathrm{min})$ & $R($ times $/ \mathrm{min})$ & $\mathrm{BP}(\mathrm{mmHg})$ & $\mathrm{WBC}(\mathrm{cells} / \mathrm{L})$ & $N(\%)$ & $L(\%)$ & $\mathrm{CRP}(\mathrm{mg} / \mathrm{L})$ \\
\hline Case 1 & 36.6 & 106 & 18 & $157 / 98$ & 4.0 & 57.6 & 29.0 & 5.1 \\
Case 2 & 37.0 & 91 & 20 & $155 / 81$ & 8.6 & 79.8 & 10.3 & 111.2 \\
Case 3 & 37.5 & 103 & 23 & $127 / 71$ & 17.6 & 93.6 & 3.6 & 89.0 \\
Case 4 & 37.3 & 92 & 18 & $113 / 79$ & 2.5 & 63.3 & 29.2 & 3.6 \\
\hline $\begin{array}{l}\text { Reference } \\
\text { range }\end{array}$ & $35.7-37.5$ & $60-100$ & $12-20$ & $(90-140) /$ & $4.0 \times 10^{9}-10.0 \times 10^{9}$ & $50.0-70.0$ & $20.0-40.0$ & $0.0-8.0$ \\
\hline
\end{tabular}

$T$ : temperature; $P$ : pulse; $R$ : respiratory; BP: blood pressure; WBC: white blood cell; $N$ : percent of neutrophils; $L$ : percent of lymphocytes; CRP: C-reactive protein. $1 \mathrm{mmHg}=133.3 \mathrm{~Pa}$

consolidations under the pleura in the basal segment of both lower lungs with bronchial ventilation signs (Fig. 1a), which were in accordance with radiographic features of COVID-19. After repeatedly asking about her epidemiological history and telephone inquiries to her colleagues, the medical staff discovered that her colleague had recently lived in Wuhan and had recently had a fever and respiratory symptoms. We immediately isolated the patient, and SARS-CoV-2 RNA was quickly detected by rRT-PCR. The positive fluorescence detection by rRT-PCR from the COVID-19 patient is shown in Fig. 2.

Case 2: A 56-year-old woman began to appear feverish one week ago, with the highest body temperature of $38.6^{\circ} \mathrm{C}$, accompanied by cough, expectoration, chest tightness, shortness of breath, and muscle soreness. After $3 \mathrm{~d}$ of antibiotic treatment in a local hospital, her body temperature returned to normal, but she still had a cough, expectoration, chest tightness, and other discomforts. Later, the patient was transferred to the emergency department of our hospital on Jan. 23, 2020, with her husband and herself firmly denying any history of living in or traveling to the epidemic areas in the previous month and denying exposure to other patients with COVID-19. The results of the physical exam and laboratory tests are shown in Table 1 . The physical exam revealed moist rales in both lower lungs. A lung CT scan showed multiple flaky and patchy groundglass shadows with unclear edges and uneven densities, mainly in the peripheral and posterior lungs (Fig. 1b), which are in accordance with the radiographic features of COVID-19. We immediately isolated the patient, and she quickly tested positive for SARS-CoV-2 RNA by rRT-PCR. After repeated, stern inquiries into her epidemiological history, the medical and disease control staff found that her husband had a short-term stay in Wuhan half a month prior.
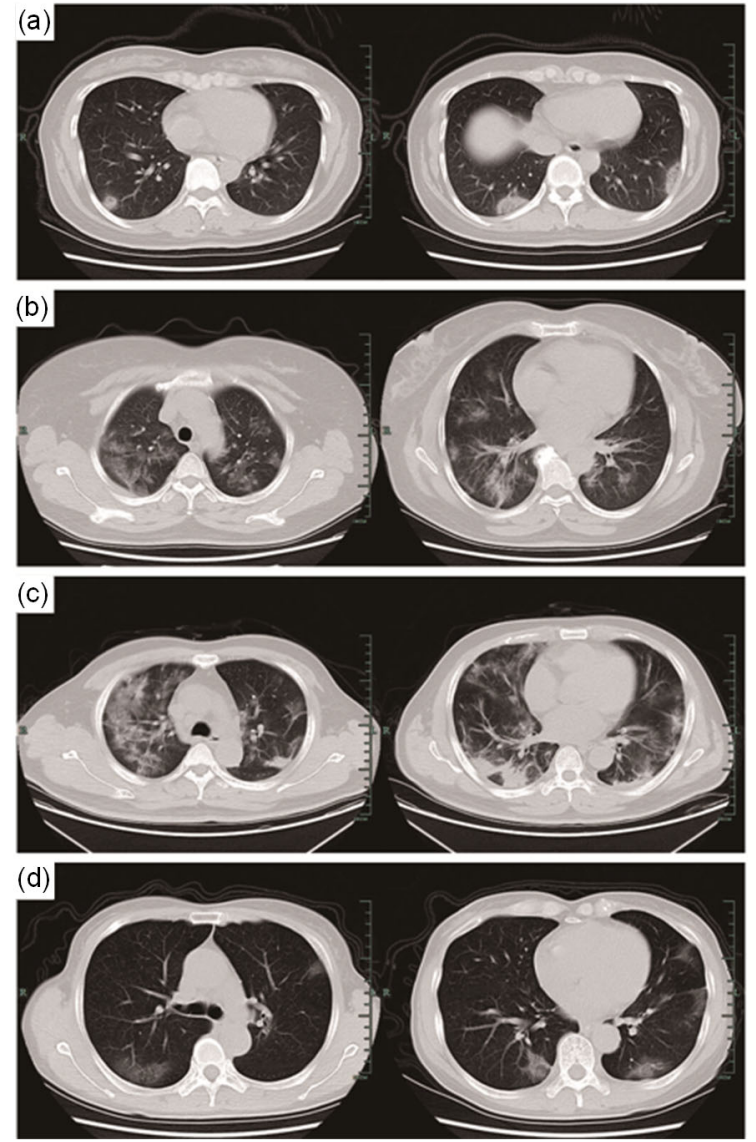

Fig. 1 Imaging studies of lung CT scans

(a) A nodular, dense shadow in the subpleural basal segment of the right lower lobe with halo sign and patchy consolidations under the pleura in the basal segment of both lower lungs with bronchial ventilation signs. (b) Multiple flaky and patchy ground-glass shadows with unclear edges and uneven densities, mainly in the peripheral and posterior lungs. (c) Multiple patchy ground-glass shadows and striplike consolidations with unclear boundaries in both lungs, especially under the pleura. (d) Multiple small patchy and flaky ground-glass shadows in the left upper lung (mainly posterior and lingual) and posterior upper lobe and middle lobe (mainly medial) of the right lung, especially under the pleura. CT: computed tomography 


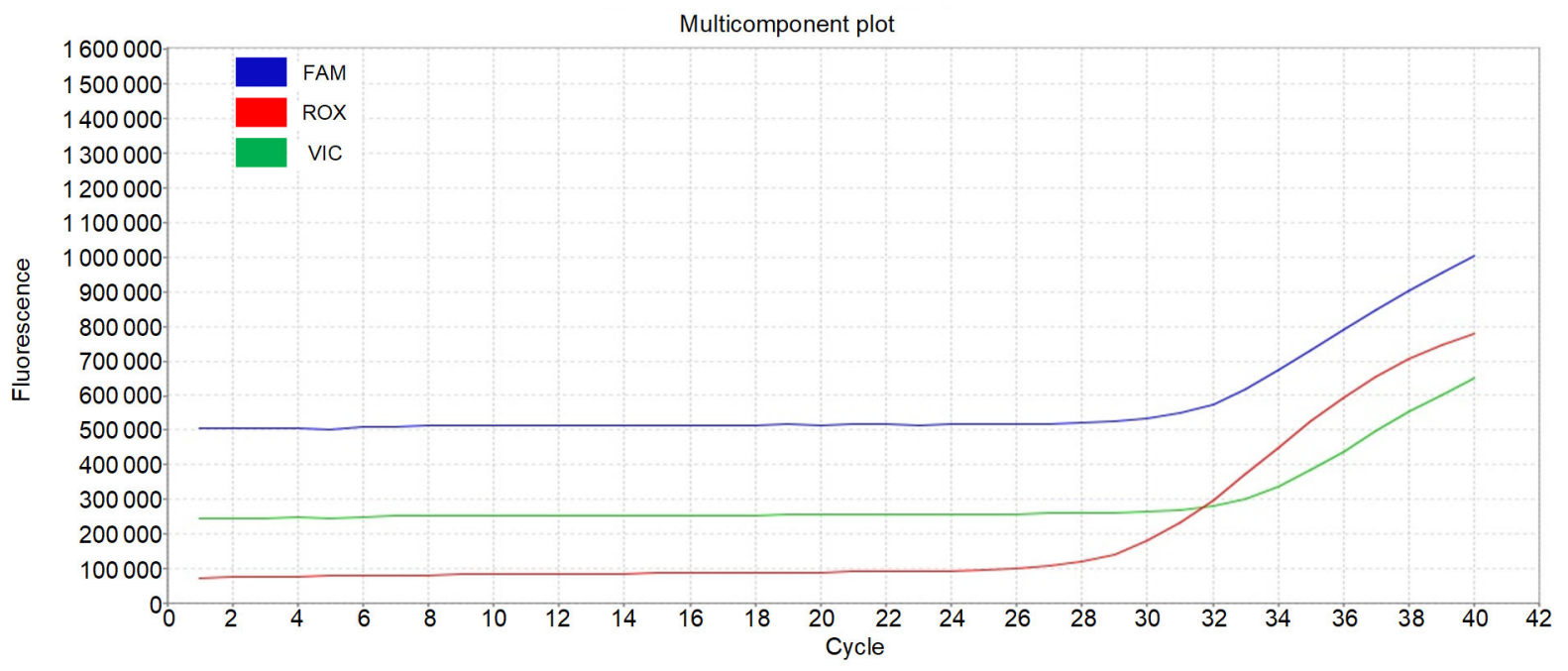

Fig. 2 Fluorescence detection of positive specimens from COVID-19 patients by rRT-PCR

A specimen is considered positive for COVID-19 if all COVID-19 markers (ORF1ab, IQC, N) cycle threshold growth curves cross the threshold line. COVID-19: coronavirus disease 2019; rRT-PCR: real-time reverse-transcriptase polymerase chain reaction; FAM: a reporter fluorescein dye targeting ORF1ab; ROX: a reporter fluorescein dye targeting IQC; VIC: a reporter fluorescein dye targeting $\mathrm{N}$

Case 3: A 48-year-old man presented with chills and a fever $11 \mathrm{~d}$ prior to diagnosis, with the highest body temperature of $38.4^{\circ} \mathrm{C}$, which was accompanied by systemic soreness and fatigue but no cough, expectoration, chest tightness, or shortness of breath; he then presented with a cough containing a little white sputum, chest tightness, and shortness of breath $2 \mathrm{~d}$ before visiting the fever clinic. The chest CT scan at a local hospital showed scattered infection in both lungs, indicating viral pneumonia, and his condition gradually worsened even after symptomatic treatments. Later, the patient visited the fever clinic at our hospital on Jan. 29, 2020, with him and his family denying a history of living in or traveling to the epidemic areas in the previous month and denying exposure to other patients with COVID-19. The results of the physical exam and laboratory tests upon admission are shown in Table 1. Physical exam revealed fine moist rales in both lungs. The results of the influenza A+B RNA assay and the SARS-CoV-2 RNA assay (both by real-time fluorescence RT-PCR) were negative. After being treated symptomatically, the patient was transferred to the emergency department on the second day. Then, a second lung CT scan showed multiple patchy ground-glass shadows and strip-like consolidations with unclear boundaries in both lungs, especially under the pleura (Fig. 1c), which are in accordance with radiographic features of COVID-19.
We immediately isolated the patient, and SARS-CoV-2 RNA with unknown epidemiological history was detected using another sample.

Case 4: A 64-year-old woman presented with chills and a fever $3 \mathrm{~d}$ after flying back to Hangzhou from Malaysia $10 \mathrm{~d}$ ago, with the highest body temperature of $38.7^{\circ} \mathrm{C}$, accompanied by cough with a little sputum, headache, and systemic soreness but no chest tightness or shortness of breath. She visited a fever clinic $5 \mathrm{~d}$ before diagnosis in our emergency department, and the results of laboratory tests were unremarkable. The result of the influenza A+B RNA assay (rRT-PCR) was negative, and no significant change in the lung $\mathrm{CT}$ scan was observed. Considering drug treatment ineffective, the patient visited the emergency department of our hospital on Feb. 1, 2020. The results of the physical exam and laboratory tests upon admission are shown in Table 1. Physical examination revealed no dry or moist rales either lung. A second lung CT scan showed multiple small patchy and flaky ground-glass shadows in the left upper lung (mainly posterior and lingual) and posterior upper lobe and middle lobe (mainly medial) in the right lung, especially under the pleura (Fig. 1d), which are in accordance with radiographic features of COVID-19. We immediately isolated the patient, and SARS-CoV-2 RNA was detected by rRT-PCR. After repeated inquiries into her medical history, the patient may have 
been exposed to COVID-19 during a recent flight (public transportation) with no obvious exposure to other patients.

Our clinical research protocol was reviewed and approved by the Ethical Committee of the First Affiliated Hospital, School of Medicine, Zhejiang University, Hangzhou, China (No. IIT20200025A).

\section{Discussion}

The epidemiological history of exposure to patients from epidemic areas or with communicable diseases, in addition to etiology detection, is of great importance for the screening and diagnosis of novel infectious diseases in early stages. Most COVID-19 patients are confirmed to have a clear epidemiological history, whereas a few present under an uncertain epidemiological history ( $\mathrm{Lu}$ et al., 2020). The four representative patients in this study were all confirmed during the second line of medical defense, the emergency department of our hospital, because of different reasons.

Case 1: The patient was not consciously, i.e., passively infected, which is likely the most common epidemiological history. Therefore, during epidemics of infectious diseases, standard personal protection, such as wearing a mask and washing hands frequently, is necessary to protect ourselves and others.

Case 2: The patient deliberately concealed her epidemiological history, committing a minor crime. This outbreak of COVID-19, due to its unknown clinical features and related rumors, has resulted in significant mental pressure and problems for the public, including anxiety, depression, and fear, which directly affects our behaviors, including an increase in self-preservation, reduction of altruistic behaviors, and even deception (Soeteman et al., 2006; Inoue and Yamaoka, 2017; Bao et al., 2020).

Case 3: The complicated diagnosis of this patient, without clear epidemiological history, showed a false negative result in the first SARS-CoV-2 RNA assay. These results appear to be mainly due to unqualified specimens (especially from throat swabs), degraded virus RNA during the detection process with a small load, poor quality of kits, and operator mistakes (To et al., 2020; Xie et al., 2020). Therefore, in clinical practice, highly suspect cases should be verified through repeated tests on deep or diversified specimens (To et al., 2020).

Case 4: The patient had a recent history of flying (public transportation), which might also be considered as an epidemiological history of exposure to the public. Therefore, less travel to public places, less use of public transportation, and less gathering are necessary during the infectious disease outbreaks along with the use of effective protection, thereby helping reduce the incidence of disease.

It is important to note that the imaging of patients with COVID-19 has obvious characteristics, especially lung $\mathrm{CT}$, which shows multiple small patchy shadows and interstitial changes, mainly in the peripheral lungs at the early stage, and then multiple ground-glass opaque infiltrates in both lungs (Lin et al., 2020; Pan et al., 2020). Severe patients can present with signs of pulmonary consolidations, but pleural effusions are rare. The four representative patients diagnosed in the emergency department in this study all relied on prompt lung CT examinations. Because of the possibility of false negative results for SARS-CoV-2 RNA by rRT-PCR from the patients' nasopharyngeal swabs, chest (or lung) CT is of great importance in screening and diagnosis of patients with no definite epidemiological history or who may have concealed contact history in the epidemic area.

As several patients had no clear epidemiological history or fever but might still be infectious during the screening process of COVID-19, protection of the medical staff in the emergency department is particularly important. The emergency department of our hospital developed a series of protective measures after the outbreak of the COVID-19 epidemic: (1) the medical staff in the emergency and outpatient departments are in strict accordance with the second level of protection (wearing level B personal protective equipment); (2) a single consulting/examination room is provided for each doctor on duty; during office visits, the patients are required to keep a suitable distance from one another; (3) patients with respiratory symptoms are treated in a separate room, and then that room is disinfected after each visit; (4) if a patient is diagnosed with COVID-19, the video information Editore (DS-IC0120-1F, DS-IA0100-2X/L, DS-2CD7027FWD/F-A, Hikvision Digital Technology Co., Ltd., Hangzhou, China) is retrieved from the control room of our hospital immediately to 
determine close contacts of the patient. These contacts based on Editore are then put into quarantine or under close observation according to the actual situation. So far, no medical staff or other staff have been infected in our emergency department.

In summary, for the screening and diagnosis of COVID-19, inquiry into the patients' epidemiological history requires meticulousness and patience. The medical staff should pay attention to the patients' psychological state, and scaled evaluation should be carried out if necessary. For patients with uncertain epidemiological history, lung CT examination has clear clinical significance. At the same time, shortterm $(2-3 \mathrm{~d})$ re-examination of lung CT scans is necessary as this disease changes rapidly. Therefore, as the second line of medical defense for screening infectious diseases in hospitals, the emergency department has irreplaceable clinical value in the accurate screening and diagnosis of COVID-19.

\section{Contributors}

Qin ZHANG performed the treatment and data analysis, wrote and edited the manuscript. Jian PAN and Min-xing ZHAO performed the treatment. Yuan-qiang LU contributed the study design, data analysis, writing and editing of the manuscript. All authors have read and approved the final manuscript and, therefore, have full access to all the data in the study and take responsibility for the integrity and security of the data.

\section{Compliance with ethics guidelines}

Qin ZHANG, Jian PAN, Min-xing ZHAO, and Yuanqiang LU declare that they have no conflict of interest.

All procedures followed were in accordance with the ethical standards of the responsible committee on human experimentation (institutional and national) and with the Helsinki Declaration of 1975, as revised in 2008 (5). Informed consent was obtained from all patients for being included in the study. Additional informed consent was obtained from all patients for whom identifying information is included in this article.

\section{References}

Bao YP, Sun YK, Meng SQ, et al., 2020. 2019-nCoV epidemic: address mental health care to empower society. Lancet, 395(10224):E37-E38. https://doi.org/10.1016/S0140-6736(20)30309-3

Feng MX, Li YN, Ruan WS, et al., 2018. Predictive value of the maximum serum creatinine value and growth rate in acute paraquat poisoning patients. Sci Rep, 8(1):11587. https://doi.org/10.1038/s41598-018-29800-0

Huang CL, Wang YM, Li XW, et al., 2020. Clinical features of patients infected with 2019 novel coronavirus in Wuhan,
China. Lancet, 395(10223):497-506.

https://doi.org/10.1016/S0140-6736(20)30183-5

Inoue M, Yamaoka K, 2017. Social factors associated with psychological distress and health problems among elderly members of a disaster-affected population: subgroup analysis of a 1-year post-disaster survey in Ishinomaki area, Japan. Disaster Med Public Health Prep, 11(1):64-71. https://doi.org/10.1017/dmp.2016.147

Lin XQ, Gong ZY, Xiao ZK, et al., 2020. Novel coronavirus pneumonia outbreak in 2019: computed tomographic findings in two cases. Korean J Radiol, 21(3):365-368. https://doi.org/10.3348/kjr.2020.0078

Lu RJ, Zhao X, Li J, et al., 2020. Genomic characterisation and epidemiology of 2019 novel coronavirus: implications for virus origins and receptor binding. Lancet, 395(10224): 565-574. https://doi.org/10.1016/s0140-6736(20)30251-8

$\mathrm{Lu}$ YQ, 2018. HIV and paraquat poisoning: fighting fire with fire? J Zhejiang Univ-Sci B (Biomed \& Biotechnol), 19(2): 168-170. https://doi.org/10.1631/jzus.B1700567

Pan YY, Guan HX, Zhou SC, et al., 2020. Initial CT findings and temporal changes in patients with the novel coronavirus pneumonia (2019-nCoV): a study of 63 patients in Wuhan, China. Eur Radiol, online. https://doi.org/10.1007/s00330-020-06731-x

Soeteman RJH, Yzermans CJ, Kerssens JJ, et al., 2006. The course of post-disaster health problems of victims with pre-disaster psychological problems as presented in general practice. Fam Pract, 23(3):378-384. https://doi.org/10.1093/fampra/cml009

To KKW, Tsang OTY, Yip CCY, et al., 2020. Consistent detection of 2019 novel coronavirus in saliva. Clin Infect Dis, online. https://doi.org/10.1093/cid/ciaa149

Wang HR, Pan J, Shang AD, et al., 2017. Time-dependent haemoperfusion after acute paraquat poisoning. Sci Rep, $7: 2239$

https://doi.org/10.1038/s41598-017-02527-0

WHO (World Health Organization), 2020. Coronavirus. https://www.who.int/health-topics/coronavirus [Accessed on Jan. 21, 2020].

Xie XZ, Zhong Z, Zhao W, et al., 2020. Chest CT for typical 2019-nCoV pneumonia: relationship to negative RT-PCR testing. Radiology, online. https://doi.org/10.1148/radiol.2020200343

$\mathrm{Xu} \mathrm{YG,} \mathrm{Lu} \mathrm{YQ,} \mathrm{2019.} \mathrm{Systematic} \mathrm{review} \mathrm{and} \mathrm{meta-analysis}$ of the efficacy and safety of immunosuppressive pulse therapy in the treatment of paraquat poisoning. $J$ Zhejiang Univ-Sci B (Biomed \& Biotechnol), 20(7):588-597. https://doi.org/10.1631/jzus.B1800640

Zhang Q, Wu WZ, Lu YQ, et al., 2012. Successful treatment of patients with paraquat intoxication: three case reports and review of the literature. J Zhejiang Univ-Sci B (Biomed \& Biotechnol), 13(5):413-418. https://doi.org/10.1631/jzus.B1200008 
Zhu JY, Pan J, Lu YQ, 2015. A case report on indirect transmission of human rabies. $J$ Zhejiang Univ-Sci B (Biomed \& Biotechnol), 16(11):969-970. https://doi.org/10.1631/jzus.B1500109

Zhu N, Zhang DY, Wang WL, et al., 2020. A novel coronavirus from patients with pneumonia in China, 2019. $N$ Engl J Med, 382(8):727-733.

https://doi.org/10.1056/NEJMoa2001017

\section{中文概要}

题 目: 中国急诊科在新型冠状病毒肺炎篮查和诊断中的 临床价值

目 的: 通过对浙江大学医学院附属第一医院急诊科筛查
出的 4 例新型冠状病毒肺炎 (新冠肺炎) 病例进 行分析, 探讨急诊科在新冠肺炎精准篮查和诊断 中的作用。

创新点: 分析新型冠状病毒肺炎的致病机制、传播方式和 临床特征; 总结急诊科医护人员的自身防护和诊 断策略。

方 法: 回顾性分析我院急诊科笁查出的 4 例新型冠状病 毒肺炎病例, 总结急诊科的临床价值。

结 论: 在新冠肺炎的筛查和诊断体系中, 中国急诊科发 挥了不可替代的作用。急诊科医护人员应充分做 好自身防护，对患者进行细致、耐心、谨慎地病 史采集和全面的体格检查, 并关注患者的心理状 态, 有效应用肺部计算机断层扫描 (CT)、核酸 检测等检测方法, 做好新冠肺炎筛查工作。

关键词: 新型冠状病毒肺炎; SARS-CoV-2; 急诊科 